\title{
Integrin $\alpha 4$ is involved in the regulation of glioma-induced motility of bone marrow mesenchymal stem cells
}

\author{
JIANG-CHUN MA ${ }^{1 *}$, PENG CHENG $^{1 *}$, YI HU ${ }^{2}$, YI-XUE XUE ${ }^{3,4}$ and YUN-HUI LIU ${ }^{2}$ \\ ${ }^{1}$ Department of Neurosurgery, The First Hospital of China Medical University, Shenyang, Liaoning 110001; \\ ${ }^{2}$ Department of Neurosurgery, Shengjing Hospital of China Medical University, Shenyang, Liaoning 110004; \\ ${ }^{3}$ Neurobiology, ${ }^{4}$ Institute of Pathology and Pathophysiology, College of Basic Medicine, \\ China Medical University, Shenyang, Liaoning 110001, P.R. China
}

Received March 9, 2015; Accepted May 8, 2015

DOI: $10.3892 /$ or.2015.4012

\begin{abstract}
Bone marrow mesenchymal stem cells (BMSCs) have the ability of migrating towards glioma tissue. However, this migratory behavior remains to be elucidated. The aim of this study was to define the role of integrin $\alpha 4$ in the motility of BMSCs towards glioma. The role of integrin $\alpha 4$ in the migration of BMSCs towards glioma was evaluated using an in vitro migration assay with the application of a specific integrin $\alpha 4$-blocking antibody. The effect of glioma conditioned medium (CM) on the integrin $\alpha 4$ expression level of BMSCs was assessed by RT-PCR, immunocytochemistry and western blot analysis. BAY11-7082, LY294002, SB203580, PD98059 and SP600125 were used to investigate the role of NF- $\kappa B$, PI3K, p38 MAPK, MEK and JNK in the above process. In addition, the role of NF- $\mathrm{\kappa B}$ in the tropism of BMSCs towards glioma was also evaluated using the in vitro model. The migration of BMSCs towards glioma CM was attenuated by blocking integrin $\alpha 4$. The stimulation of glioma CM increased integrin $\alpha 4$ expression of BMSCs. Furthermore, the inhibition of NF- $\mathrm{KB}$ and PI3K decreased the glioma-induced integrin $\alpha 4$ upregulation on BMSCs. Inhibition of NF- $\mathrm{KB}$ decreased the number of migrating BMSCs towards gliomas. Glioma cells induced the migration of BMSCs by promoting the expression of integrin $\alpha 4$. NF- $\mathrm{kB}$ and PI3K contributed to the signal transduction of this process. Similar to PI3K, NF- $\mathrm{KB}$ is associated with the regulation of BMSCs migration toward glioma. Thus, these results may be useful to elucidate the mechanism involved in the glioma-induced migration of BMSCs.
\end{abstract}

Correspondence to: Dr Peng Cheng, Department of Neurosurgery, The First Hospital of China Medical University, 155 Nanjingbei Street, Heping, Shenyang, Liaoning 110001, P.R. China

E-mail: chengpengcmu@hotmail.com; chengpengcmu@sina.com

*Contributed equally

Key words: mesenchymal stem cells, glioma, integrin $\alpha 4$, cell migration, bone marrow

\section{Introduction}

As the most common type of malignant primary neoplasms in the central nervous system, gliomas are characterized by their highly aggressive and infiltrative growth pattern (1). Gliomas are easy to be recurrent even after extensive surgical resection combined with radio- and chemotherapy treatment $(2,3)$. In recent years, targeted gene therapy against gliomas has attracted increasing attention (4-6). The application of gene therapy has led to concerns being raised regarding the selection of the proper vector for gene therapy against glioma. Bone marrow mesenchymal stem cells (BMSCs) are a type of adult stem cells isolated from bone marrow. Due to their directional migration abilities towards gliomas after transplantation, they have been applied as the vectors of gene therapy against gliomas (7-9). However, the molecular mechanisms responsible for this migratory behavior have not been fully explained and require further investigation.

Integrins are a group of heterodimeric transmembrane proteins that participate in facilitating the adhesion of cells to their surroundings, such as extracellular matrix (10). Recent findings demonstrated that integrins are involved in the regulation of cell migration, such as lymphocytes infiltration in inflammation and tumors $(11,12)$. Integrin $\alpha 4$ is a subunit of vascular cell adhesion molecule-1 (VCAM-1) receptor, very late antigen-4 (VLA-4). The binding interaction between integrin $\alpha 4$ and its ligand VCAM-1 is critical to the migration of B lymphocytes through the fibroblast barrier (13). Furthermore, it has been reported that the interaction between integrins and VCAM-1 is associated with the infiltration of $\mathrm{T}$ lymphocytes through the blood-brain barrier in the central nervous system inflammation (14). In addition, VLA-4 and VCAM-1 are involved in the migration of melanoma cells across activated endothelial cell layers (15). Our previous studies have shown that VCAM-1 contributed to the migration of BMSCs toward gliomas (16). Additionally, integrin $\alpha 4$ is important, not only as the ligand of VCAM-1, but also as the vital component in the formation of adhesion plaque (17). Based on these observations, we hypothesized that integrin $\alpha 4$ participates in mediating the tropism of BMSCs towards gliomas. To examine the functional role of integrin $\alpha 4$, we applied conditioned medium (CM) of glioma cells to incubate 
BMSCs and found that the increased motility of BMSCs was integrin $\alpha 4$-dependent. In addition, we analyzed the impact of glioma CM on the integrin $\alpha 4$ expression of BMSCs. The results showed that glioma $\mathrm{CM}$ elevated the expression of integrin $\alpha 4$ in both the mRNA and protein levels. Furthermore, we studied the role of PI3K, NF-кB, MEK, p38 MAPK and JNK in the upregulation of integrin $\alpha 4$ induced by glioma $\mathrm{CM}$. The results showed that NF- $\mathrm{KB}$ and PI3K contributed to the signal transduction of glioma-induced integrin $\alpha 4$ upregulation on BMSCs.

\section{Materials and methods}

Animals. Healthy female SD rats (4-6-week-old) were used for the isolation and culture of BMSCs. The rats were obtained from the Laboratory Animal Center of China Medical University (Liaoning, China). The animal procedures were approved by the Animal Care and Use Committee of China Medical University and conducted in accordance with the National Institute of Health Guide for the Care and Use of Laboratory Animals.

Reagents and antibodies. The primary and secondary antibodies used in this study were: monoclonal anti-integrin $\alpha 4$ for blocking (Abcam, Hong Kong, China); anti-integrin $\alpha 4$ for immunofluorescence and western blot analysis (Cell Signaling Technology, Danvers, MA, USA); anti-GAPDH (Santa Cruz Biotechnology, Dallas, TX, USA); FITC-labeled anti-rat CD34, CD45, CD73, CD90 and CD105 (Bioss, Beijing, China); and the secondary antibodies for western blot analysis, HRP-conjugated anti-rabbit IgG and HRP-conjugated anti-mouse IgG (both from ZsBio, Beijing, China). An enhanced chemiluminescence kit (ECL) was purchased from Thermo Fisher Scientific (Waltham, MA, USA). Fetal bovine serum (FBS) and Dulbecco's modified Eagle's medium of low glucose (L-DMEM) were both obtained from Gibco (Waltham, MA, USA).

Isolation and culture of BMSCs. The BMSCs were isolated and expanded by their characteristics of adherence to plastic as previously described $(18,19)$. Briefly, the rats were euthanized by $\mathrm{CO}_{2}$ inhalation following cervical dislocation after intraperitoneal injection of $10 \%$ chloral hydrate $(3.5 \mathrm{ml} / \mathrm{kg})$. Bilateral tibias and femurs were excised by aseptic procedures. Bone marrow cells were flushed out from the bones using L-DMEM containing 10\% FBS. Then the cells were transferred into a $\mathrm{T} 75 \mathrm{~cm}^{2}$ flask and incubated at $37^{\circ} \mathrm{C}$ with $5 \% \mathrm{CO}_{2}$. The non-adherent cells were discarded by changing the media after $48 \mathrm{~h}$. When the adherent cells grew to $90 \%$ confluence, the cells were detached by $0.25 \%$ trypsin and passaged at a ratio of 1:2. The cells at passage 3 were applied to the subsequent experiments.

Differentiation assay. Osteogenic, adipogenic and chondrogenic induction were performed as previously described $(20,21)$. To demonstrate the multilineage differentiation capacity of the cells used in this study, P3 BMSCs $\left(1 \times 10^{5}\right.$ cells in $1 \mathrm{ml}$ medium) were seeded in 6-well plates. After the cell grew to $80 \%$ confluence, the culture media were changed to osteogenic (dexamethasone $0.1 \mu \mathrm{M}, \beta$-glycerol phosphatase
$10 \mathrm{mM}$ and ascorbate $50 \mu \mathrm{M}, 10 \% \mathrm{FBS})$ (19), adipogenic (IBMX $0.5 \mathrm{mM}$, dexamethasone $1 \mu \mathrm{M}$, insulin $10 \mu \mathrm{g} / \mathrm{ml}$ and indomethacin $200 \mu \mathrm{M}, 10 \%$ FBS) (19) and chondrogenic differentiation media (insulin $6.25 \mu \mathrm{g} / \mathrm{ml}$, TGF- $\beta 210 \mathrm{ng} / \mathrm{ml}$ and 2-ascorbic acid $50 \mathrm{nM}$ ) (20), separately. The media were refreshed every 3 days. After two weeks, the cells were fixed with $4 \%$ paraformaldehyde (PFA) and then stained with $0.1 \%$ alizarin red S, $3 \%$ oil red $\mathrm{O}$ or $1 \%$ alcian blue, respectively, to show the formation of mineral, lipid deposits or glycosaminoglycans. The images were captured under an upright microscope (IX71; Olympus, Tokyo, Japan).

Culture of glioma cell line and the preparation of glioma CM. The rat C6 glioma cell line and human U87 and U251 glioblastoma cell lines, were purchased from the Chinese Academy of Medical Sciences. The cells were cultured and maintained in L-DMEM with 10\% FBS under the same conditions of temperature and humidity as BMSCs. The CM of C6, U251 and U87 glioma cells was prepared as previously described (22). Briefly, when the cells grew to $80 \%$ confluence in the T75 flask, the complete media were removed and the cells were washed twice with PBS and followed by incubation with $10 \mathrm{ml}$ serum-free L-DMEM for $24 \mathrm{~h}$. The cells were collected and centrifuged at $1,000 \mathrm{x}$ g for $10 \mathrm{~min}$ to remove cell debris. The aliquots of the supernatant were stored at $-80^{\circ} \mathrm{C}$ until required.

Flow cytometric analysis. Flow cytometry was carried out as previously described (18), to determine the expression of $\mathrm{CD}$ markers in the isolated cells. Third-passage BMSCs were collected and fixed in $70 \%$ ethanol at $4^{\circ} \mathrm{C}$ for $30 \mathrm{~min}$. The cells were incubated with FITC-labeled anti-CD34, CD45, CD73, CD90 and CD105 antibodies for $2 \mathrm{~h}$, washed and resuspended with PBS and then analyzed by FACScan flow cytometer (Becton-Dickinson, San Jose, CA, USA).

In vitro migration assay. Transwell cell culture chambers of $8-\mu \mathrm{m}$ pore size (Corning Costar, MA, USA) were applied to assess the role of integrin $\alpha 4$ in the glioma-induced motility of BMSCs. After BMSCs were trypsinized, the cells were collected and resuspended in serum-free L-DMEM at a density of $5 \times 10^{5} / \mathrm{ml}$. Then, $200 \mu \mathrm{l}$ of BMSCs suspension was added into the upper chambers. Glioma CM was placed into the lower chambers. The blocking antibody of integrin $\alpha 4$ was added into the upper chamber to a final concentration of $20 \mu \mathrm{g} / \mathrm{ml}$ to neutralized integrin $\alpha 4$ bioactivity. After the cells were co-cultured for $24 \mathrm{~h}$, the cells migrating to the underside of the membrane were stained with Giemsa and the number of cells was evaluated as previously described (16). To assess the role of NF- $\mathrm{KB}$ in the migration of BMSCs towards glioma, BAY11-7082 (NF-kB inhibitor; Enzo, New York, NY, USA) was applied to incubate BMSCs at a final concentration of $5 \mu \mathrm{M}$ during the stimulation of glioma $\mathrm{CM}$.

Cell proliferation assay. A cell proliferation assay was used to investigate the effect of glioma CM incubation on the growth of BMSCs. An in vitro growth assay was used to prevent the proliferation of BMSCs affecting the evaluation. BMSCs were seeded in 96 -well plates at a density of $5 \times 10^{3}$ cells/well in $100 \mu 1 \mathrm{~L}-D M E M$ containing $10 \%$ FBS with five replicate wells for each group. The cells were incubated overnight, and 
when the cells adhered to the plastic, the culture media were changed to C6 CM, U251 CM and U87 CM, respectively. After incubation of 0 and 24 h, $20 \mu 1$ MTS solution (Promega, Beijing, China) was added into the wells and the cells were then incubated for $1.5 \mathrm{~h}$ in the dark. The absorbance at $490 \mathrm{~nm}$ was measured by a multi-channel microplate reader (SpectraMax M5).

RNA isolation, semi-quantitative-PCR and quantitative PCR ( $q P C R$ ). BMSCs were treated with C6, U87 and U251 CM for $24 \mathrm{~h}$, respectively. Total RNA was isolated by TRIzol reagent (Life Technologies, Danvers, MA, USA), following the manufacturer's instructions. The concentration of RNA was determined by a NanoDrop 2000 (Thermo Fisher Scientific). The Takara RNA PCR kit (AMV) ver. 3.0 was used for reverse transcription (Takara, Dalian, China). For routine PCR, the primer sequences of were: integrin $\alpha 4,5$-ATCTAGTTTTTA CACACAGGATTT-3' (forward) and, 5'-TGTCAATGTCGC CAAGATT-3' (reverse) (23); GAPDH, 5'-CCGTATCGGACG CCTGGTTA-3' (forward) and, 5'-TCTCGCTCCTGGAAG ATGGTG-3' (reverse). The length of the amplified fragment was 533 bp for integrin $\alpha 4$ and 207 bp for GAPDH. The PCR reaction was carried out under the following conditions: denaturation at $94^{\circ} \mathrm{C}$ for $30 \mathrm{sec}$, annealing at $52^{\circ} \mathrm{C}$ for $30 \mathrm{sec}$, and extension at $72^{\circ} \mathrm{C}$ for $1 \mathrm{~min}$, and 35 cycles. GAPDH was applied as an internal control. The PCR product was identified using $1 \%$ agarose gel electrophoresis containing Genefinder (Zeesan, Xiamen, China). The ratio of the integrated density values (IDV) of the integrin $\alpha 4$ and GAPDH gene was then calculated. qPCR was performed on a TP800 Real-Time PCR system using the SYBR Premix Ex TaqTM II kit (both from Takara) and specific integrin $\alpha 4$ primers as follows: 5'-CGGCACGCT GTTTGGCTACT-3' (forward) and, 5'-ATCGCCCCAGGA TTGACCAC-3' (reverse). GAPDH served as an internal control. The primers for GAPDH were the same as those for routine PCR. Amplification was performed for 40 cycles as follows: $30 \mathrm{sec}$ at $95^{\circ} \mathrm{C}$, and for each cycle $5 \mathrm{sec}$ at $95^{\circ} \mathrm{C}$ for denaturation and $30 \mathrm{sec}$ at $60^{\circ} \mathrm{C}$ for annealing. Relative mRNA quantification of integrin $\alpha 4$ to GAPDH was determined by the $2^{-\Delta \Delta \mathrm{Ct}}$ method. In addition, to investigate the roles of PI3K, NF- $\kappa \mathrm{B}$, MEK, p38 MAPK and JNK in the changes of the integrin $\alpha 4$ expression of BMSCs induced by glioma CM, LY294002 (PI3K inhibitor, $30 \mu \mathrm{M}$; Cell Signaling Technology), BAY11-7082 (5 $\mu \mathrm{M})$, PD98059 (MEK inhibitor, $10 \mu \mathrm{M}$ ), SB203580 (p38MAPK inhibitor, $10 \mu \mathrm{M}$ ) and SP600125 (JNK inhibitor, $10 \mu \mathrm{M}$; all from Enzo) were applied to incubate BMSCs 30 min before, and for the duration of glioma CM stimulation, separately. Serum-free L-DMEM was used as a negative control.

Immunocytochemistry. For immunofluorescence, BMSCs at $70 \%$ confluence were trypsinized and seeded onto cover slips coated with $1.5 \%$ gelatin. After $48 \mathrm{~h}$, the cells were treated with glioma CM for $24 \mathrm{~h}$, followed by fixation with $4 \%$ PFA. The cells were blocked with 5\% BSA for $2 \mathrm{~h}$ and incubated with monoclonal rabbit anti-rat integrin $\alpha 4$ antibody at $4{ }^{\circ} \mathrm{C}$ overnight. TRITC conjugated anti-rabbit secondary antibody was then used for visualization. Nuclei were counterstained with DAPI. Fluorescent photomicrographs were captured using an upright microscope (DP71; Olympus). Identical filters, objectives and acquisition parameters were used for each experiment.

Western blot analysis. BMSCs were incubated with C6, U251 and U87 CM as described above. The cell lysate of each group was prepared in lysis buffer containing protease and phosphatase inhibitor cocktail on ice (Sigma Aldrich). The sample protein concentrations were determined using a Bicinchoninic Acid Protein Assay kit (Beyotime Biotechnology, Shanghai, China). An equal amount of protein lysates ( $25 \mu \mathrm{g} /$ lane) were fractionated on $8 \%$ SDS-polyacrylamide gel and transferred to PVDF membrane. Subsequently, the membranes were blocked with $5 \%$ skimmed milk for $2 \mathrm{~h}$ and then treated with anti-integrin $\alpha 4$ and anti-GAPDH antibodies at $4{ }^{\circ} \mathrm{C}$ overnight, respectively. The protein bands were visualized with an ECL Detection system. The IDVs were measured by the Fluor Chen 2.0 software and GAPDH protein served as an internal control.

Statistical analysis. Triplicate replications were performed for each experiment. Data were analyzed by SPSS 19.0 and presented as mean \pm SD. Statistical differences between two groups were assessed using the Student's t-test. One-way analysis of variance test (ANOVA) followed by Dunnett's post test were used to compare differences among multiple groups. $\mathrm{P}<0.05$ was considered statistically significant.

\section{Results}

The characterization of BMSCs immunophenotype and multipotent differentiation capacities. The isolated cells demonstrated the ability of adherence to plastic and exhibited spindle-like shape (Fig. 1A, upper panel). After being passaged, the cells grew to typical fibroblast colonies (Fig. 1A, lower panel). The results obtained by the flow cytometry showed that the isolated cells of passage 3 were positive for CD73 (98.7\%), CD90 (99.1\%) and CD105 (98.3\%), and negative for CD34 (1\%) and CD45 (0.966\%) (Fig. 1B). Furthermore, following incubation with differentiation medium for 2 weeks, the cells showed positive staining for mineral nodules formation, lipid deposits and glycosaminoglycans. These results indicated that the cells had the capacity of differentiation into osteoblasts, adipocytes and chondroblasts in vitro (Fig. 1C). Based on these results, the cells used in this study were consistent with the definition of BMSCs as previously described (19-21).

Integrin $\alpha 4$ is involved in the regulation of BMSCs motility promoted by glioma. Our previous findings showed that the stimulation of glioma CM induced the directional migration of BMSCs and VCAM-1 functioned as an important adhesion molecule in the glioma-induced migration of BMSCs (16). Since VCAM-1 is the counter ligand of VLA-4 and VLA-4 is an integrin dimer composed of integrin $\alpha 4$ and $\beta 1$, we investigated the relationship between integrin $\alpha 4$ and the glioma-stimulated migration of BMSCs. The results of the in vitro migration assay showed that the number of migrating BMSCs towards glioma CM was significantly decreased with the addition of an integrin $\alpha 4$-blocking antibody, when compared with the control group (Fig. 2A and B). These results suggested that integrin $\alpha 4$ contributed to the glioma-induced 

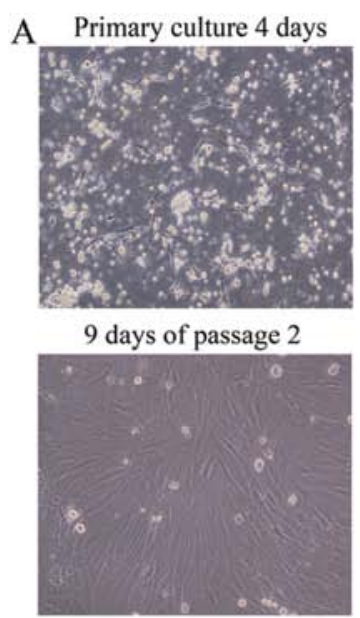

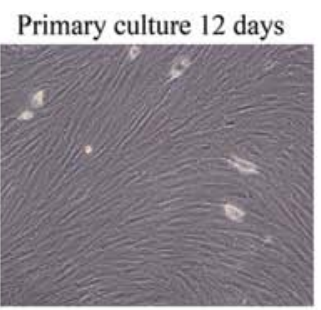

9 days of passage 3

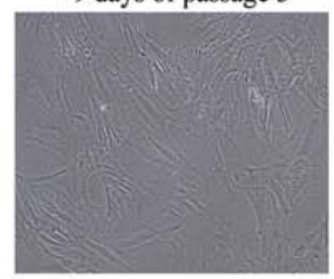

B

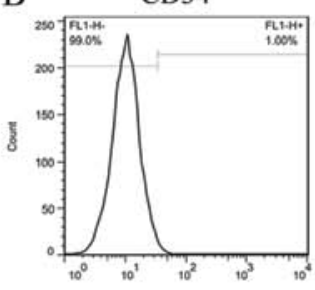

F.1.
CD45

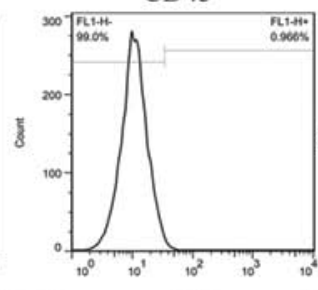

CD90

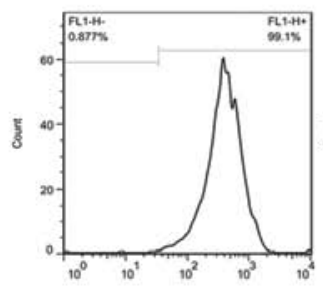

Flit

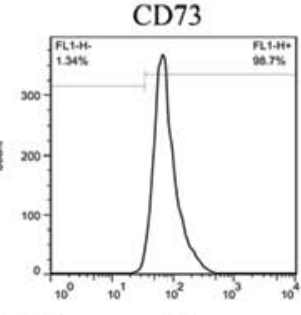

CD105

Fin
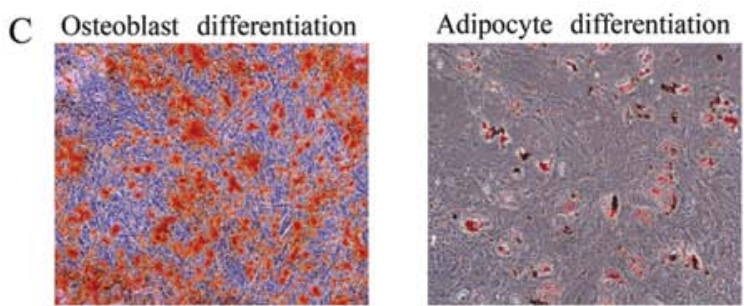

Chondroblast differentiation
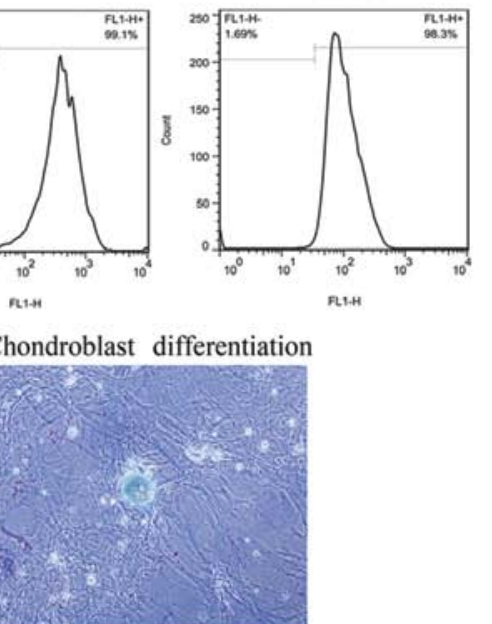

Figure 1. Morphology, immunophenotype and multiple differentiations of BMSCs. (A) Upper panel: representative image of BMSCs on the 4th and 12th days of primary culture; lower panel: representative image of BMSCs at passages 2 and 3. (B) Flow cytometry results of CD34, CD45, CD73, CD90 and CD105 on BMSCs at passage 3. (C) Representative images of the in vitro differentiation assay on BMSCs at passage 3. Left panel: osteoblast differentiation; middle panel: adipocyte differentiation; right panel: chondroblast differentiation. Original magnification, x200.

A

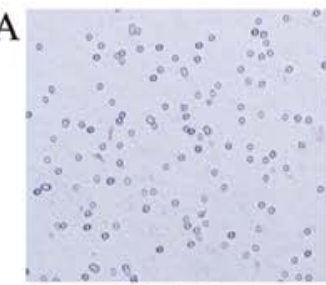

Control

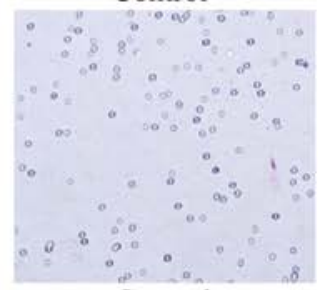

Control

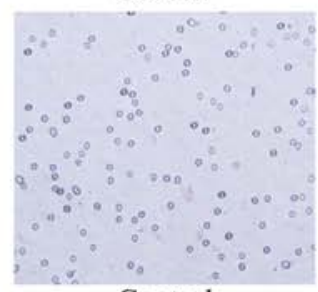

Control

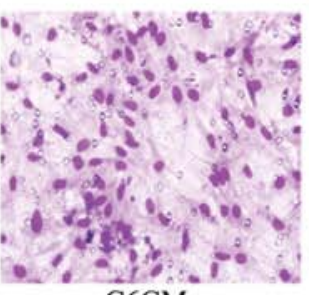

C6CM

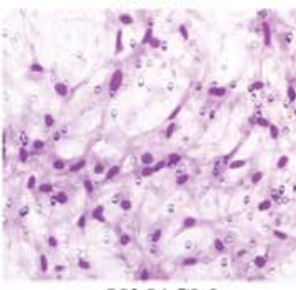

$\mathrm{U} 251 \mathrm{CM}$

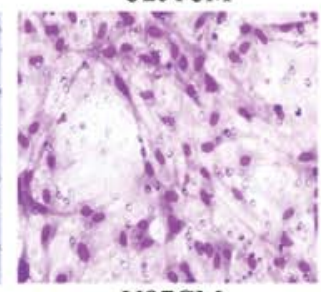

U87CM

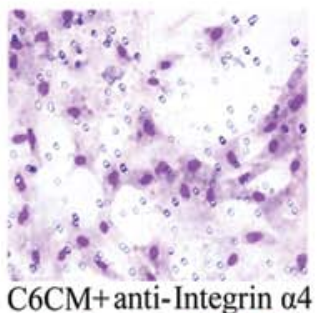

$\mathrm{C} 6 \mathrm{CM}+$ anti-Integrin $\alpha 4$

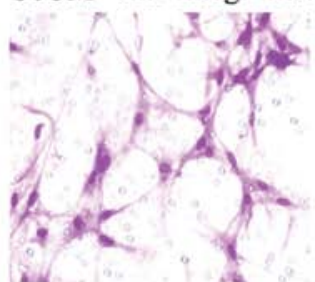

$\mathrm{U} 251 \mathrm{CM}+$ anti-Integrin $\alpha 4$

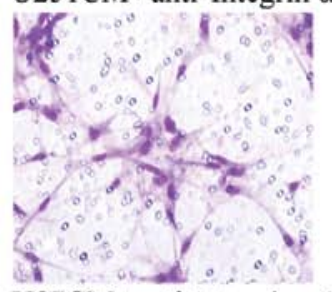

$\mathrm{U} 87 \mathrm{CM}+$ anti-Integrin $\alpha 4$
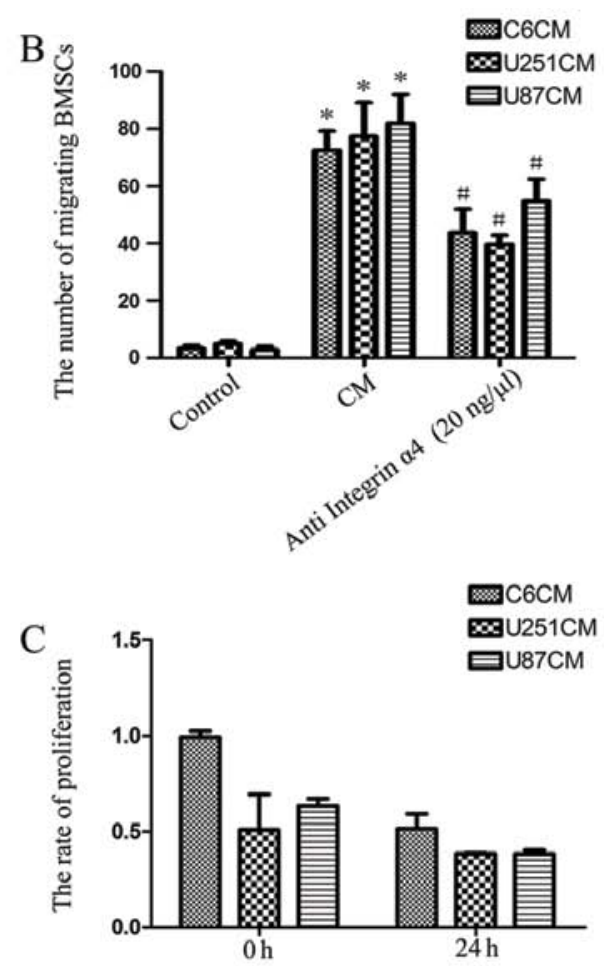

Figure 2 . Role of integrin $\alpha 4$ in the glioma-induced BMSCs migration. (A) Images showing the results of the in vitro migration assay after stimulation of glioma CM for $24 \mathrm{~h}$. Left panel: control group (incubated with serum-free L-DMEM); middle panel: glioma CM group (incubated with glioma CM); right panel: glioma $\mathrm{CM}+$ anti-integrin $\alpha 4$ (co-incubated with glioma CM and an anti-integrin $\alpha 4$ blocking antibody at $20 \mu \mathrm{g} / \mathrm{ml}$ ). The glioma CM in the lower chamber significantly increased the migration of BMSCs, and this migration can be inhibited by the addition of the anti-integrin $\alpha 4$ blocking antibody. Original magnification, $\mathrm{x} 400$. (B) Statistical analysis of the in vitro migration assay. ${ }^{*} \mathrm{P}<0.01$ vs. control group, $\mathrm{n}=6 .{ }^{*} \mathrm{P}<0.01$ vs. glioma $\mathrm{CM}$ group, $\mathrm{n}=6$. (C) Graph indicating the impact of 24-h glioma CM incubation on the proliferation of BMSCs. The incubation of glioma CM did not increase the proliferation of BMSCs, $\mathrm{n}=3$. 
A

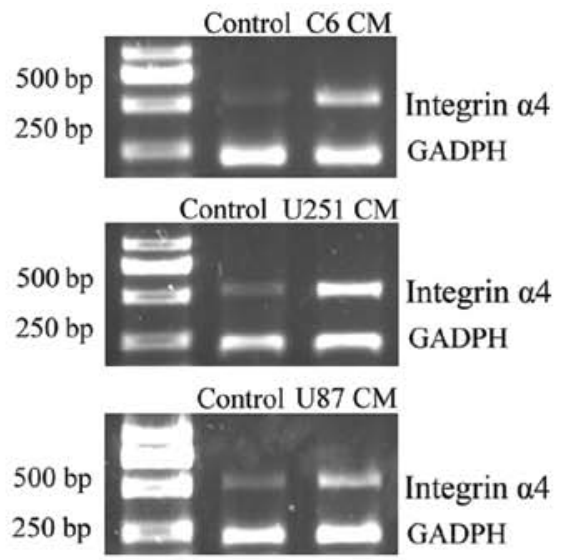

C
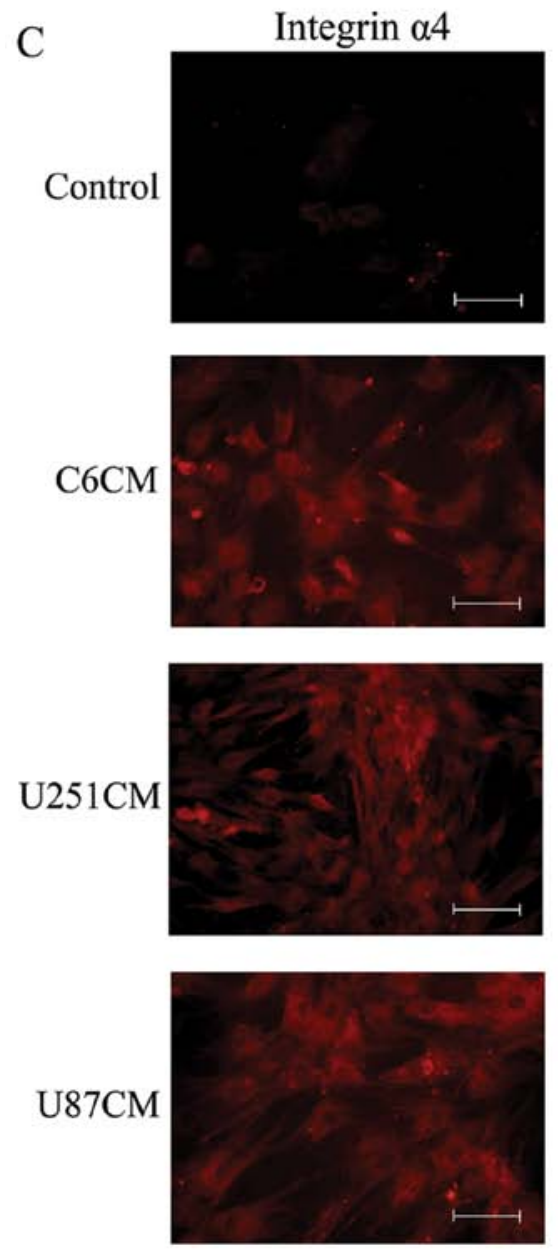

B

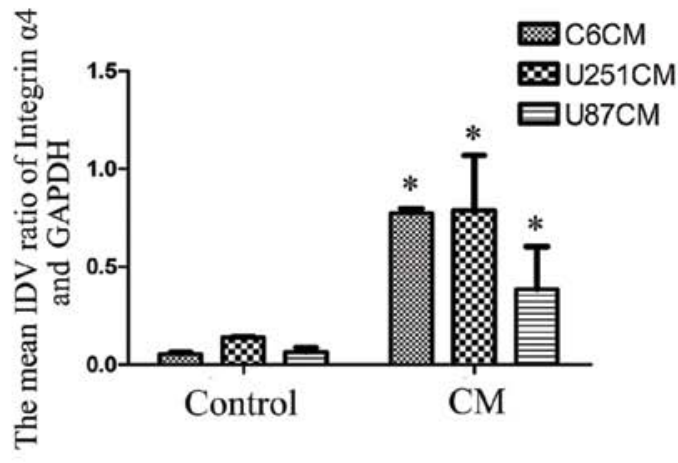

DAPI

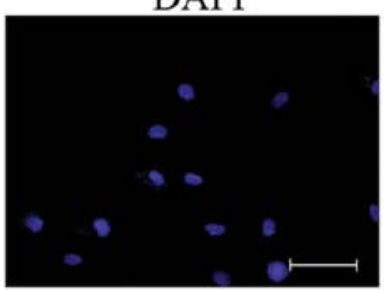

Integrin $\alpha 4+\mathrm{DAPI}$
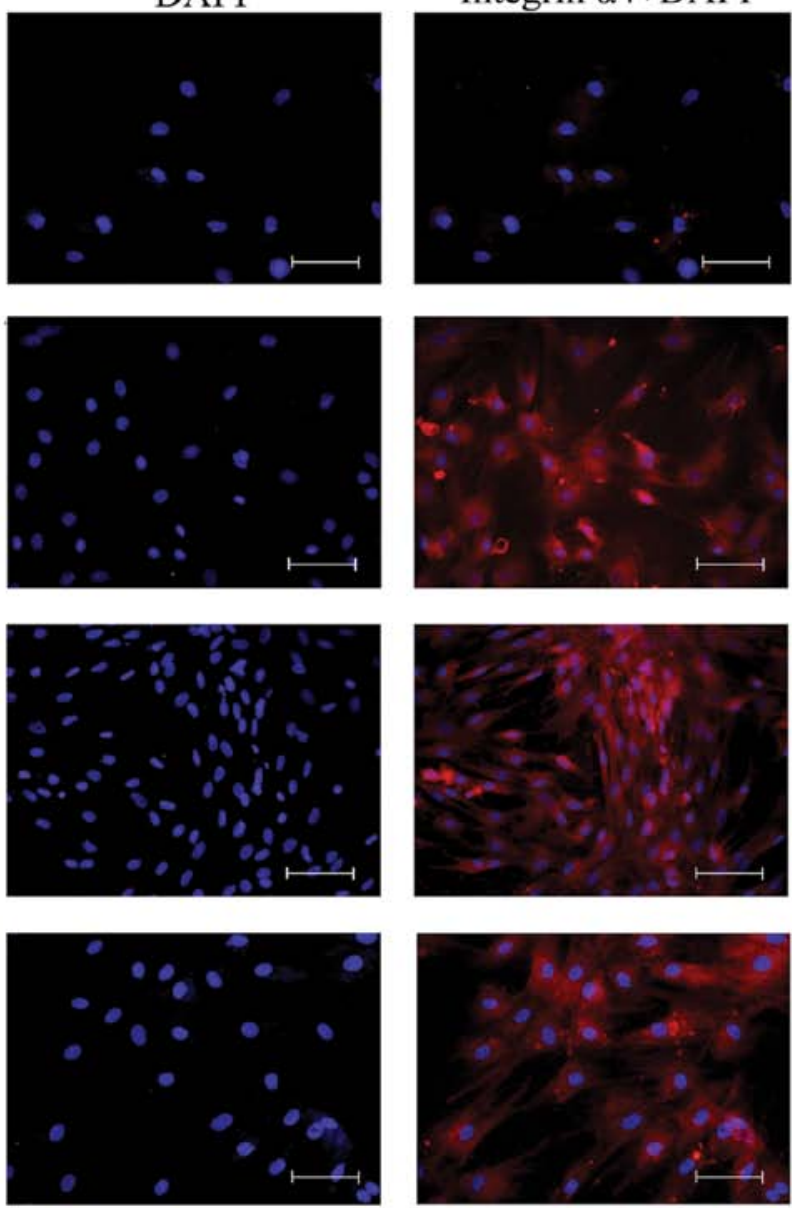

Figure 3. Impact of glioma CM on integrin $\alpha 4$ expression of BMSCs. The results show that the incubation of C6, U87 and U251 CM increased the expression level of integrin $\alpha 4$ on BMSCs, compared with serum-free L-DMEM. (A) Representative images of semi-quantitative PCR. (B) Statistical analysis of RT-PCR. "P $<0.01$ vs. control group, $\mathrm{n}=6$. (C) Representative results of immunocytochemistry. Scale bars, $100 \mu \mathrm{m}$.

motility of BMSCs. We also examined the growth-stimulating activity of glioma CM on BMSCs by MTS assay to prevent the influence of glioma CM on BMSC proliferation using an in vitro migration assay. The results showed that the incubation of glioma CM for $24 \mathrm{~h}$ did not significantly increase the number of BMSCs in comparison with the control group (L-DMEM) (Fig. 2C).

Integrin a 4 expression on BMSCs is elevated by the stimulation of glioma CM. We studied the impact of glioma $\mathrm{CM}$ on the expression of integrin $\alpha 4$ of BMSCs. In order to assess the effect of glioma CM on the integrin $\alpha 4$ expression of BMSCs, BMSCs were treated with C6, U87 and U251 CM for $24 \mathrm{~h}$, and RT-PCR and immunofluorescence were performed to determine the mRNA and protein expression of integrin $\alpha 4$. As shown in Fig. 3, incubation with glioma CM for $24 \mathrm{~h}$ significantly increased the expression of integrin $\alpha 4$ at the mRNA (Fig. 3A and B) and protein levels (Fig. 3C).

$N F-\kappa B$ and PI3K are involved in mediating the glioma-induced upregulation of integrin $\alpha 4$ on BMSCs. To clarify the regulatory mechanisms associated with the glioma-induced integrin $\alpha 4$ 


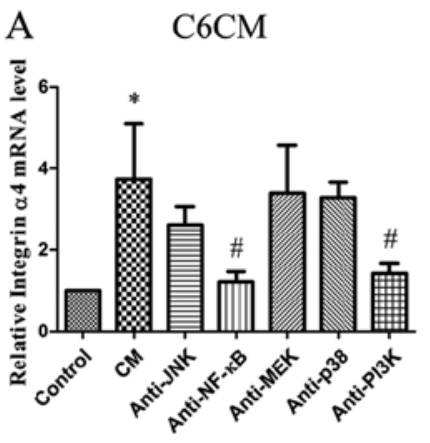

B

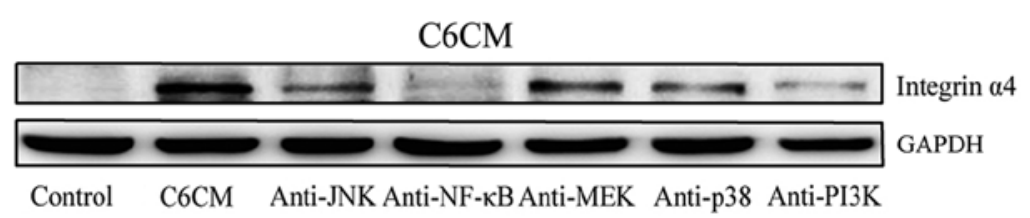

$\mathrm{U} 251 \mathrm{CM}$

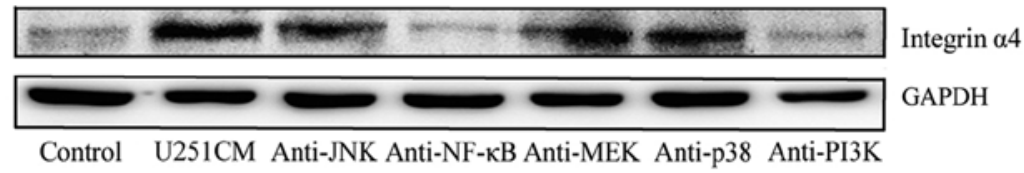

Control U251CM Anti-JNK Anti-NF-кB Anti-MEK Anti-p38 Anti-PI3K

$\mathrm{U} 251 \mathrm{CM}$

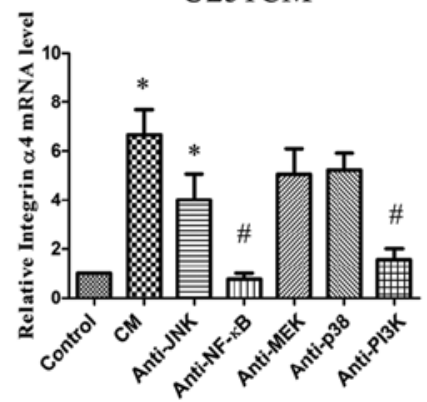

$\mathrm{C}$
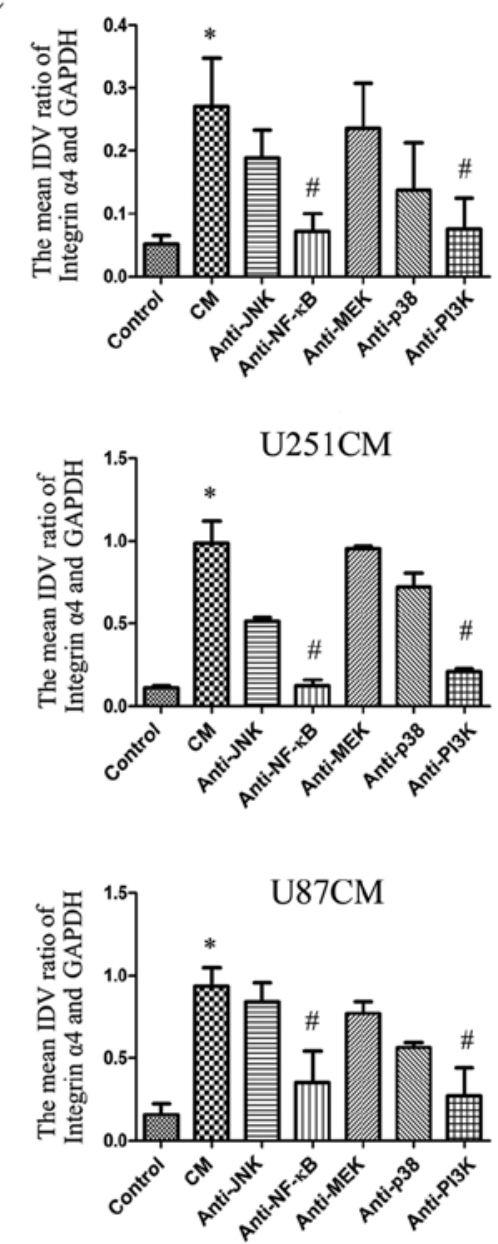

Figure 4. Involvement of NF- $\mathrm{KB}$ and PI3K in the glioma-induced upregulation of integrin $\alpha 4$. The glioma-induced elevated expression of integrin $\alpha 4$ on BMSCs was inhibited by the application of BAY11-7082, an NF- $\mathrm{kB}$ inhibitor, and LY294002, a PI3K inhibitor, showing the important role of NF- $\mathrm{KB}$ and PI3K in this process. (A) Graph showing the qPCR results on the integrin $\alpha 4$ mRNA expression change after the incubation of glioma CM. GAPDH served as a control. ${ }^{*} \mathrm{P}<0.01$ vs. control group, $\mathrm{n}=4 .{ }^{~} \mathrm{P}<0.01$ vs. glioma $\mathrm{CM}$ group, $\mathrm{n}=4$. (B) Representative images of western blotting demonstrating integrin $\alpha 4$ protein expression on BMSCs with the stimulation of glioma CM. GAPDH was used as a control. (C) Statistical analysis of western blot results. "P<0.01 vs. control group, $\mathrm{n}=3$. $\mathrm{P}<0.01$ vs. glioma $\mathrm{CM}$ group, $\mathrm{n}=3$.

upregulation of BMSCs, NF- $\mathrm{B}$ inhibitor BAY11-7082, PI3K inhibitor LY294002, p38 MAPK inhibitor SB203580, JNK inhibitor SP600125 and MEK inhibitor PD98059 were applied in our study. As shown in Fig. 4, the data demonstrated that the glioma CM-induced upregulation of integrin $\alpha 4$ was significantly reduced with the application of BAY11-7082 and LY294002. By contrast, there was no significant inhibition obtained by the addition of SB203580, SP600125 and PD98059 (Fig. 4). These results suggested that NF- $\kappa$ B and PI3K participated in the intracellular signal transduction of glioma CM-induced upregulation of integrin $\alpha 4$.
Inhibition of $N F-\kappa B$ decreases the motility of BMSCs promoted by glioma. We previously demonstrated that PI3K played a crucial role in the glioma-induced migration of BMSCs (16). As demonstrated above, integrin $\alpha 4$ contributed to mediating the glioma-induced migration of BMSCs, and NF- $\mathrm{BB}$ and $\mathrm{PI} 3 \mathrm{~K}$ were key signaling molecules in glioma-induced upregulation of integrin $\alpha 4$. Thus, we hypothesized that NF- $\kappa \mathrm{B}$ is also associated with the regulation of glioma-induced migration of BMSCs. To assess the role of $N F-\kappa B$ in the glioma-induced migration of BMSCs, an in vitro migration assay was performed. As shown in Fig. 5, the results of the 


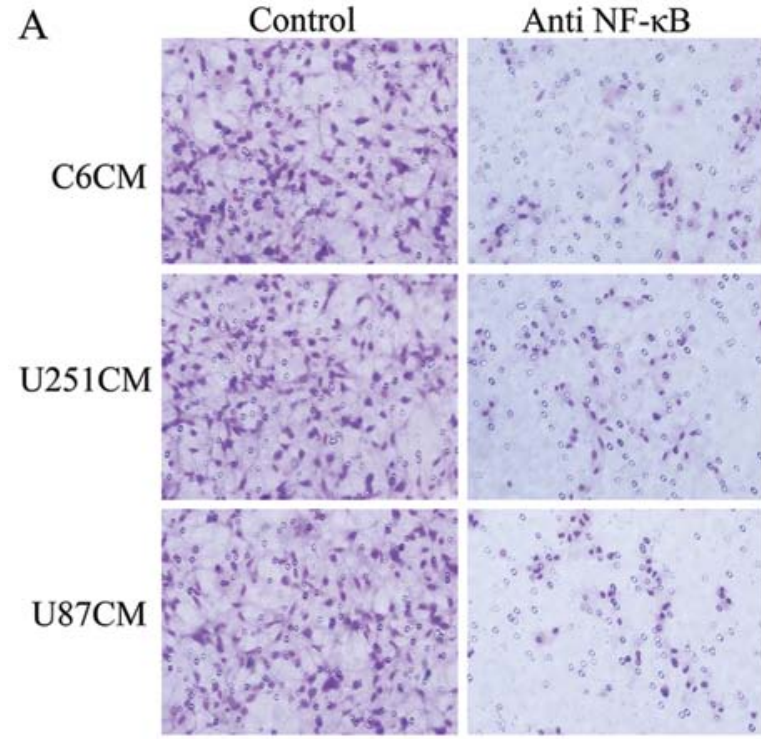

B

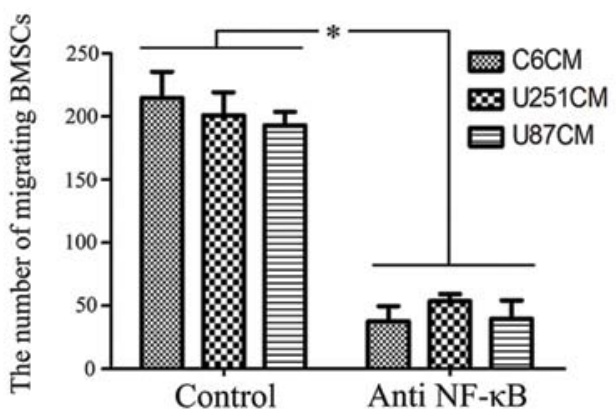

Figure 5. Role of NF- $\kappa b$ in the motility of BMSCs promoted by glioma. (A) Images showing the results of the in vitro migration assay after adding BAY11-7082 into the upper chamber of the Transwell inserts during the $24 \mathrm{~h}$ stimulation of glioma CM. Left panel: glioma CM group (incubated with glioma CM); right panel: BAY11-7082 group (5 $\mu \mathrm{M} / \mathrm{ml})$. Original magnification, $\mathrm{x} 400$. (B) Statistical analysis of the in vitro migration assay. ${ }^{*} \mathrm{P}<0.05$ vs. glioma CM group, $\mathrm{n}=3$.

in vitro migration showed that the treatment of $5 \mu \mathrm{M}$ BAY117082 as mentioned above significantly decreased the motility of BMSCs under the stimulation of C6, U87 and U251 glioma CM ( $\mathrm{P}<0.05$ for C6 CM, U251 CM, and U87 CM group), which indicated that $\mathrm{NF}-\kappa \mathrm{B}$ played an important role in the glioma-induced migration of BMSCs.

\section{Discussion}

In the present study, we confirmed that integrin $\alpha 4$ plays a crucial role in the migration of BMSCs towards glioma. We also provided evidence for the upregulation of integrin $\alpha 4$ on BMSCs with the stimulation of glioma $\mathrm{CM}$ and the regulatory role of $\mathrm{NF}-\kappa \mathrm{B}$ and $\mathrm{PI} 3 \mathrm{~K}$ in this process. Moreover, $\mathrm{NF}-\kappa \mathrm{B}$ contributed to mediating the glioma-induced migration of BMSCs.

As a type of adult stem cells, BMSCs have increasingly attracted attention due to their multiple differentiation abilities and clinical feasibility. Previous studies have shown that BMSCs migrated directionally to glioma tissue $(9,16)$. Accordingly, some studies applied BMSCs as the vector for targeted gene therapy against gliomas and obtained encouraging data $(8,9)$. However, the mechanisms for the tropism of BMSCs towards glioma have yet to be fully elucidated and require further investigation.

As a member of the integrin family, integrin $\alpha 4$, also known as CD49d, is a multifunctional adhesive factor. Integrin $\alpha 4$ is important in the physiological development of various types of cells, and contributes to the migration of neuron in embryonic development $(15,24,25)$. Furthermore, integrin $\alpha 4$ is a subunit of the VCAM-1 receptor, VLA-4, and it has been reported that the interaction between VCAM-1 and VLA-4 enhanced the migration of human melanoma cells across activated endothelial cell layers (15). In a previous study, glioma cells have been demonstrated to be able to promote the migration of BMSCs by increasing VCAM-1 expression of BMSCs (16). Moreover, a previous report confirmed the crucial role of the integrin $\alpha 4$ /VCAM-1 pathway in leukocyte migration across the blood-brain barrier (26). A previous in vivo study has shown that integrin $\alpha 4$ /VCAM-1 interactions induced the firm adhesion formation of $\mathrm{T}$ cell/blood-brain barrier (14). Based on these observations, we hypothesized that integrin $\alpha 4$ may be involved in the regulation of glioma-induced motility of BMSCs and further investigated its role in this process. We found that the addition of an integrin $\alpha 4$-blocking antibody in the upper chamber of Transwell inserts significantly decreased the number of BMSCs migrating to the lower chamber. This result suggests that integrin $\alpha 4$ was involved in the regulation of glioma-induced motility of BMSCs. Furthermore, we incubated BMSCs with glioma CM of C6, U251 and U87 cells and measured the expression levels of integrin $\alpha 4$ prior to and after the incubation. The results confirmed that the incubation of glioma CM increased integrin $\alpha 4$ expression of the mRNA and protein levels. Our previous studies $(16,18)$ found that the CM of glioma promoted the expression of VCAM-1, similar to integrin $\alpha 4$. Therefore, it is reasonable to conclude that glioma may promote the migration of BMSCs by increasing the expression of integrin $\alpha 4$. Additionally, we suggest that VCAM-1 and integrin $\alpha 4$ might interact with their own ligands in the extracellular matrix, respectively, which promote the migration in a 2-fold effect, which is similar to tumor cells such as U87MG $(27,28)$.

To determine the signaling pathway associated with the glioma-induced changes of the integrin $\alpha 4$ expression of BMSCs, we investigated the roles of PI3K, NF- $\mathrm{B}, \mathrm{P} 38$ MAPK, MEK and JNK in the regulation of integrin $\alpha 4$ expression on BMSCs in this study. qPCR and western blotting revealed that glioma-induced integrin $\alpha 4$ upregulation was notably inhibited by BAY11-7082 and LY294002, but not by SB203580, SP600125 and PD98059. Thus, NF-кB and PI3K pathways were associated with the intracellular signaling transduction of integrin $\alpha 4$ upregulation induced by gliomas. Moreover, the results of the in vitro migration assay confirmed that the co-incubation of NF- $\kappa \mathrm{B}$ inhibitor BAY11-7082 with C6, U251 and U87 glioma CM decreased the glioma-induced migration of BMSCs. Since our previous report showed that PI3K contributed to the signaling transduction in the glioma-induced migration of BMSCs (16), it is reasonable to infer that integrin $\alpha 4$ possibly regulates the glioma-induced motility of BMSCs through the NF- $\kappa \mathrm{B}$ and PI3K pathways.

In conclusion, our results have shown that integrin $\alpha 4$ participated in mediating the glioma-induced motility of 
BMSCs. We have also determined that glioma cells increased the expression of integrin $\alpha 4$ of the mRNA and protein levels via an NF- $\kappa \mathrm{B}$ - and PI3K-dependent signal transduction. $\mathrm{NF}-\mathrm{\kappa B}$ is one of the signaling molecules that contributed to the signal transduction of the glioma-induced migration of BMSCs. These results may be useful for future studies on the mechanism of the glioma-induced migration of BMSCs.

\section{Acknowledgements}

The present study was supported by the Natural Science Foundation of China, under the contract nos. 30901781 and 81302191, and the Liaoning Science and Technology Plan Projects no. 2012225014.

\section{References}

1. Olar A and Aldape KD: Using the molecular classification of glioblastoma to inform personalized treatment. J Pathol 232: 165-177, 2014.

2. Jones TS and Holland EC: Standard of care therapy for malignant glioma and its effect on tumor and stromal cells. Oncogene 31 1995-2006, 2012.

3. Wen PY and Kesari S: Malignant gliomas in adults. N Engl J Med 359: 492-507, 2008.

4. Sasportas LS, Kasmieh R, Wakimoto H, Hingtgen S, van de Water JA, Mohapatra G, Figueiredo JL, Martuza RL, Weissleder R and Shah K: Assessment of therapeutic efficacy and fate of engineered human mesenchymal stem cells for cancer therapy. Proc Natl Acad Sci USA 106: 4822-4827, 2009.

5. Murphy AM and Rabkin SD: Current status of gene therapy for brain tumors. Transl Res 161: 339-354, 2013.

6. Bexell D, Svensson A and Bengzon J: Stem cell-based therapy for malignant glioma. Cancer Treat Rev 39: 358-365, 2013.

7. Bexell D, Gunnarsson S, Tormin A, Darabi A, Gisselsson D, Roybon L, Scheding S and Bengzon J: Bone marrow multipotent mesenchymal stroma cells act as pericyte-like migratory vehicles in experimental gliomas. Mol Ther 17: 183-190, 2009.

8. Kim SM, Woo JS, Jeong CH, Ryu CH, Lim JY and Jeun SS: Effective combination therapy for malignant glioma with TRAIL-secreting mesenchymal stem cells and lipoxygenase inhibitor MK886. Cancer Res 72: 4807-4817, 2012.

9. Nakamizo A, Marini F, Amano T, Khan A, Studeny M, Gumin J, Chen J, Hentschel S, Vecil G, Dembinski J, et al: Human bone marrow-derived mesenchymal stem cells in the treatment of gliomas. Cancer Res 65: 3307-3318, 2005.

10. Cheng $M$ and Qin G: Progenitor cell mobilization and recruitment: SDF-1, CXCR4, $\alpha 4$-integrin, and c-kit. Prog Mol Biol Transl Sci 111: 243-264, 2012.

11. Haanstra KG, Hofman SO, Lopes Estêvão DM, Blezer EL, Bauer J, Yang LL, Wyant T, Csizmadia V, 't Hart BA and Fedyk ER: Antagonizing the $\alpha 4 \beta 1$ integrin, but not $\alpha 4 \beta 7$, inhibits leukocytic infiltration of the central nervous system in rhesus monkey experimental autoimmune encephalomyelitis. J Immunol 190: 1961-1973, 2013.

12. Takeichi T, Mocevicius P, Deduchovas O, Salnikova O, Castro-Santa E, Büchler MW, Schmidt J and Ryschich E: $\alpha \mathrm{L} \beta 2$ integrin is indispensable for $\mathrm{CD} 8^{+} \mathrm{T}$-cell recruitment in experimental pancreatic and hepatocellular cancer. Int J Cancer 130: 2067-2076, 2012.

13. Couture P, Paradis-Massie J, Oualha N and Thibault G: Adhesion and transcellular migration of neutrophils and B lymphocytes on fibroblasts. Exp Cell Res 315: 2192-2206, 2009.
14. Engelhardt B: Molecular mechanisms involved in $\mathrm{T}$ cell migration across the blood-brain barrier. J Neural Transm 113: 477-485, 2006

15. Klemke M, Weschenfelder T, Konstandin MH and Samstag Y: High affinity interaction of integrin alpha4beta1 (VLA-4) and vascular cell adhesion molecule 1 (VCAM-1) enhances migration of human melanoma cells across activated endothelial cell layers. J Cell Physiol 212: 368-374, 2007.

16. Hu Y, Cheng P, Xue YX and Liu YH: Glioma cells promote the expression of vascular cell adhesion molecule- 1 on bone marrow-derived mesenchymal stem cells: a possible mechanism for their tropism toward gliomas. J Mol Neurosci 48: 127-135, 2012.

17. Ilina $\mathrm{O}$ and Friedl $\mathrm{P}$ : Mechanisms of collective cell migration at a glance. J Cell Sci 122: 3203-3208, 2009.

18. Hu Y, Cheng P, Ma JC, Xue YX and Liu YH: Platelet-derived growth factor BB mediates the glioma-induced migration of bone marrow-derived mesenchymal stem cells by promoting the expression of vascular cell adhesion molecule-1 through the PI3K, P38 MAPK and NF- $\mathrm{BB}$ pathways. Oncol Rep 30: 2755-2764, 2013

19. Dominici M, Le Blanc K, Mueller I, Slaper-Cortenbach I, Marini F, Krause D, Deans R, Keating A, Prockop Dj and Horwitz E: Minimal criteria for defining multipotent mesenchymal stromal cells. The International Society for Cellular Therapy position statement. Cytotherapy 8: 315-317, 2006.

20. Pittenger MF, Mackay AM, Beck SC, Jaiswal RK, Douglas R, Mosca JD, Moorman MA, Simonetti DW, Craig S and Marshak DR: Multilineage potential of adult human mesenchymal stem cells. Science 284: 143-147, 1999.

21. Nora CC, Camassola M, Bellagamba B, Ikuta N, Christoff AP, Meirelles LS, Ayres R, Margis R and Nardi NB: Molecular analysis of the differentiation potential of murine mesenchymal stem cells from tissues of endodermal or mesodermal origin. Stem Cells Dev 21: 1761-1768, 2012.

22. Birnbaum T, Roider J, Schankin CJ, Padovan CS, Schichor C, Goldbrunner R and Straube A: Malignant gliomas actively recruit bone marrow stromal cells by secreting angiogenic cytokines. J Neurooncol 83: 241-247, 2007.

23. Umesh A, Thompson MA, Chini EN, Yip KP and Sham JS: Integrin ligands mobilize $\mathrm{Ca}^{+}$from ryanodine receptor-gated stores and lysosome-related acidic organelles in pulmonary arterial smooth muscle cells. J Biol Chem 281: 34312-34323, 2006.

24. Nishiya N, Kiosses WB, Han J and Ginsberg MH: An $\alpha 4$ integrin-paxillin-Arf-GAP complex restricts Rac activation to the leading edge of migrating cells. Nat Cell Biol 7: 343-352, 2005.

25. Garmy-Susini B, Avraamides CJ, Desgrosellier JS, Schmid MC, Foubert P, Ellies LG, Lowy AM, Blair SL, Vandenberg SR, Datnow B, et al: PI3K $\alpha$ activates integrin $\alpha 4 \beta 1$ to establish a metastatic niche in lymph nodes. Proc Natl Acad Sci USA 110: 9042-9047, 2013.

26. James WG, Bullard DC and Hickey MJ: Critical role of the $\alpha 4$ integrin/VCAM-1 pathway in cerebral leukocyte trafficking in lupus-prone MRL/fas(lpr) mice. J Immunol 170: 520-527, 2003.

27. Bernhart E, Damm S, Wintersperger A, DeVaney T, Zimmer A, Raynham T, Ireson C and Sattler W: Protein kinase D2 regulates migration and invasion of U87MG glioblastoma cells in vitro. Exp Cell Res 319: 2037-2048, 2013.

28. Wang YB, Hu Y, Li Z, Wang P, Xue YX, Yao YL, Yu B and Liu YH: Artemether combined with shRNA interference of vascular cell adhesion molecule-1 significantly inhibited the malignant biological behavior of human glioma cells. PLoS One 8: e60834, 2013. 